\title{
miR-23a-3p regulates the inflammatory response and fibrosis in diabetic kidney disease by targeting early growth response 1
}

\author{
Shuyue Sheng ${ }^{1} \cdot$ Meina Zou ${ }^{1} \cdot$ Yanlin Yang ${ }^{1} \cdot$ Meiping Guan ${ }^{1} \cdot$ Shijing Ren ${ }^{1} \cdot$ Xiangyu Wang ${ }^{1} \cdot$ Ling Wang $^{1}$. \\ Yaoming Xue ${ }^{1}$
}

Received: 21 March 2021 / Accepted: 4 July 2021 / Published online: 4 October 2021 / Editor: Tetsuji Okamoto

(C) The Author(s) 2021

\begin{abstract}
Diabetic kidney disease (DKD) has become the most common cause of chronic kidney disease. Proteinuria is generally considered one of the clinical indicators of renal damage, and it is also closely related to the progression of DKD. Accumulating evidence indicates that proteinuria induces an upregulation of the expression levels of inflammatory cytokines and fibrosis markers in renal tubular epithelial cells, but the mechanism remains unclear. Previously, we showed that early growth response 1 (Egr1) played a key role in renal tubular injury. However, the upstream mechanism of Egr1 in the development of DKD is poorly understood. In this study, we found that albumin stimulation significantly increased the expression levels of Egr1, interleukin 6 (IL-6), tumor necrosis factor- $\alpha$ (TNF- $\alpha$ ), and fibronectin (FN) in HK-2 cells but decreased miR-23a-3p levels. We then identified that miR-23a-3p targeted the 3' untranslated region (UTR) of Egrl and directly suppressed the expression of Egr1. Moreover, we found that overexpression and inhibition of miR-23a-3p in HK-2 cells attenuated and promoted the expression of IL-6, TNF- $\alpha$, and FN, respectively. Additionally, Egr1 silencing reversed the inflammation and fibrosis caused by the miR-23a-3p inhibitor. Thus, we conclude that miR-23a-3p attenuates the development of DKD through Egrl, suggesting that targeting miR-23a-3p may be a novel therapeutic approach for DKD.
\end{abstract}

Keywords Diabetic kidney disease $\cdot$ miRNA $\cdot$ Early growth response $1 \cdot$ Inflammatory response $\cdot$ Renal fibrosis

\section{Introduction}

Diabetes is an important chronic disease worldwide (Liu et al. 2019). According to the International Diabetes Federation (IDF), as of 2019, there were 463 million diabetic patients worldwide (International Diabetes Federation 2019). Diabetic kidney disease (DKD) is one of the most serious complications of diabetes. In China, DKD has surpassed chronic glomerulonephritis to become the most common cause of chronic kidney disease (CKD) (Zhang et al. 2016). Proteinuria is an important marker of the occurrence and development of DKD and is also used to evaluate the efficacy of treatment for DKD. Increasing evidence has shown that proteinuria may serve as an alternative endpoint for the study of

Shuyue Sheng and Meina Zou contributed equally to this work.

Yaoming Xue

xueyaoming999@126.com

1 Department of Endocrinology and Metabolism, Nanfang Hospital, Southern Medical University, No. 1838 Guangzhou North Avenue, Guangzhou 510515, Guangdong, China
CKD (Heerspink et al. 2019). However, the specific mechanism by which proteinuria contributes to DKD progression remains unclear. Renal fibrosis is considered a vital pathogenic feature of DKD and is characterized by excessive production of extracellular matrix (ECM) proteins, including fibronectin (FN) and collagen. Moreover, an increasing number of studies have confirmed that renal tubules play a key role in DKD (Zeni et al. 2017). Tubulointerstitial fibrosis (TIF) and tubular atrophy contribute greatly to DKD (Slyne et al. 2015). Studies by our team over the past years have shown that tubular epithelial-to-mesenchymal transition (EMT) plays a significant role in promoting the progression of TIF during DKD (Jia et al. 2019; Yang et al. 2020). Several studies have shown that the inflammatory response is a key factor in the development of DKD (Eller et al. 2011; Sakai and Wada 2015). Inflammatory cytokines such as interleukin 6 (IL-6) and tumor necrosis factor- $\alpha$ (TNF- $\alpha$ ) have been shown to be involved in the progression of DKD (Sun and Kanwar 2015; Hameed et al. 2018). Inflammatory cells activate proximal renal tubular epithelial cells (PTECs) by releasing cytokines and other mediators, resulting in excessive production of ECM, which leads to renal fibrosis (Wong et al. 2018). Therefore, 
elucidation of the pathophysiological mechanisms of PTECs during the development of DKD is important.

Recently, early growth response 1 (Egr1) was shown to be involved in the progression of fibrosis via a transforming growth factor $\beta$ (TGF- $\beta$ )/Smad-dependent signaling pathway (Bhattacharyya et al. 2008). Furthermore, Egrl was activated in a renal failure model, which impairs TGF- $\beta$-dependent renal inflammation and fibrosis (Chen et al. 2006). We have also previously reported that Egrl plays an important role in renal fibrosis in DKD (Wang et al. 2015; Yang et al. 2019). In addition, Egrl was found to be strongly associated with the inflammatory response (Peng et al. 2019). However, the upstream regulatory mechanism of Egrl in the development of DKD is poorly understood.

microRNAs (miRNAs) are a group of small, highly conserved noncoding RNAs. The length of miRNAs is approximately 20-25 nucleotides (Bartel 2018). miRNAs are posttranscriptional regulators. miRNAs bind to the $3^{\prime}$ untranslated region (UTR) of target gene messenger RNAs (mRNAs), leading to rapid degradation of the target gene or inhibition of translation (Mohajeri et al. 2018). Moreover, miRNAs have been reported to bind to other regions of target mRNAs, including the 5' UTR, coding sequence, and gene promoter. In addition, some studies have demonstrated that miRNAs can activate target gene expression under certain conditions (Broughton et al. 2016). miRNAs are regarded as important regulatory factors in the development of DKD (Jia et al. 2018; Jia et al. 2019; Zha et al. 2019). miR-23a-3p is a member of the miR-23 family. Recent studies have shown that the miR23 family plays a key role in the inflammatory response and the development of diabetes (Zhu et al. 2012; Hu et al. 2017). A previous study reported that nine miRNAs, including miR$23 \mathrm{a}$, were significantly decreased in a miRNA microarray analysis of TNF- $\alpha$-treated endothelial cells (Ruan et al. 2012). However, the role of miR-23a-3p in DKD is unknown.

The purpose of this study was to determine the relationship between miR-23a-3p and Egr1 in the inflammatory response and fibrotic progression of PTECs to further elucidate the occurrence and development of DKD.

\section{Materials and methods}

Animal studies Mouse models that were successfully constructed previously by our research team were used in this study. Detailed methods of animal model construction were described in previously published literature (Hu et al. 2018). The renal tissues of these animals were frozen intact in liquid nitrogen. Briefly, 3- to 4-wk-old male C57BL/6J mice (Guangdong Medical Laboratory Animal Center) were randomly divided into 2 groups. The mice in the control group $(n=6)$ were fed a normal diet, while the mice in the DKD group $(n=6)$ were fed a high-fat diet (HFD, protein $26.2 \%$, fat $34.9 \%$, and carbohydrate $26.3 \%$ ) for $4 \mathrm{wk}$. Next, the mice in the DKD group received intraperitoneal injection of streptozotocin (STZ, $120 \mathrm{mg} / \mathrm{kg}$ in citrate buffer, $\mathrm{pH}=4.5$, MP Biomedicals, Solon, Ohio), while the control group received equal volumes of sodium citrate. The mice in the control and DKD groups were sacrificed $12 \mathrm{wk}$ after modeling. The blood glucose of the mice in the DKD group was significantly higher than that of the mice in the control group $(22.38$ $\mathrm{mmol} / \mathrm{L}$ vs. $5.65 \mathrm{mmol} / \mathrm{L})$. All of the experiments were approved by the Institutional Animal Care and Use Committee of Nanfang Hospital, Southern Medical University, Guangzhou, China.

Cell cultures and transfection The human proximal tubule cell line (HK-2) and the human embryonic kidney 293T cell line (293T) were purchased from the China Center for Type Culture Collection (Wuhan University, Wuhan, China). HK2 cells were obtained from the Cell Bank within 6 mo. 293T cells were authenticated on November 16, 2020, in Shanghai Biowing Applied Biotechnology Co. Ltd. (Shanghai, China). DNA was extracted with Axygen's Genome Extraction Kit, amplified according to a 21-STR amplification protocol, and tested for the STR locus and the sex gene amelogenin on an ABI Model 3730XL Genetic Analyzer. We confirm that all experiments were performed with mycoplasma-free cells. HK-2 cells were cultured in $5.5 \mathrm{mmol} / \mathrm{L}$ Dulbecco's modified Eagle's medium (DMEM; Gibco, Carlsbad, CA) containing $10 \%$ fetal bovine serum (FBS; Gibco, Melbourne, Australia). $293 \mathrm{~T}$ cells were cultured in $25 \mathrm{mmol} / \mathrm{L}$ DMEM (Gibco, Carlsbad, CA) with $10 \%$ FBS. All cells were grown at $37^{\circ} \mathrm{C}$ in a humidified atmosphere containing $5 \% \mathrm{CO}_{2}$. Cells were seeded at $60-70 \%$ confluence. Culture medium containing $2 \%$ FBS was used to synchronize cells before experiments. HK-2 cells were stimulated with bovine serum albumin (BSA, $10 \mathrm{mg} / \mathrm{mL}$ ) for 1 and $48 \mathrm{~h}$. Small interfering RNA targeting Egr1 (si-Egr1; RiboBio, Guangzhou, China) was used to knockdown Egr1 in HK-2 cells, while the pENTER-Egr1 plasmid (Vigene Biosciences, Shandong, China) was used to overexpress Egr1 in HK-2 cells. In addition, a miR-23a-3p mimic and a miR-23a-3p inhibitor purchased from RiboBio (Guangzhou, China) were used to knockdown and overexpress miR-23a-3p, respectively. All transfections were performed by using Lipofectamine ${ }^{\circledR} 3000$ (Invitrogen, Shanghai, China).

RNA isolation and quantitative real-time polymerase chain reaction (qRT-PCR) TRIzol (TaKaRa, Dalian, China) was used to isolate total RNA from renal tissues and HK-2 cells. The detection of RNA concentration and purity was carried out with a NanoDrop ND-1000 spectrophotometer (Thermo Fisher Scientific, Franklin, MA). mRNA reverse transcription was performed using a Takara PrimeScript RT Reagent Kit (Takara). miRNA reverse transcription was carried out using a miRcute miRNA cDNA First Strand Synthesis Kit (Tiangen, Beijing, 
China). A SYBR Green qPCR Kit (TaKaRa) and miRcute miRNA qPCR Detection Kit (Tiangen, Beijing, China) were used to determine the mRNA and miRNA expression levels in a Roche LightCycler 480II system (Roche, Basle, Switzerland). $\beta$-Actin and U6 were used as internal controls to calculate the relative expression using the $2^{-\Delta \Delta \mathrm{Ct}}$ method. The primers used are listed in Table 1.

Western blot analysis Total protein was extracted from HK-2 cells with cold RIPA lysis buffer (KeyGEN Biotech, Nanjing, China). All processes were performed on ice to avoid degradation of the protein samples. Proteins $(30 \mu \mathrm{g})$ were separated by $10 \%$ sodium dodecyl sulfate polyacrylamide gel electrophoresis (SDS-PAGE; Bio-Rad, Hercules, CA) and then transferred to polyvinylidene fluoride (PVDF) membranes (Merck Millipore, MA). The PVDF membranes were blocked in 5\% skim milk for $1 \mathrm{~h}$. Then, the membranes were incubated with primary antibodies against Egr1 (1:1000, 55117-1-AP, anti-rabbit; ProteinTech, Wuhan, China), IL-6 (1:1000, 66146-1-Ig, anti-mouse; ProteinTech), TNF- $\alpha$ (1:1000, 60291-1-Ig, anti-mouse; ProteinTech), FN (1:500, 15613-1AP, anti-rabbit; ProteinTech), and $\beta$-actin (1:1000, 60008-1Ig, anti-mouse; ProteinTech) at $4^{\circ} \mathrm{C}$ overnight. Then, the membranes were incubated with secondary antibodies (goat anti-mouse, SA00001-1, and goat anti-rabbit, SA00001-2, 1:15,000; ProteinTech) at room temperature for $1 \mathrm{~h}$. A chemiluminescence kit (Merck Millipore, Darmstadt, Germany) was used to detect the bands of the membranes in a chemiluminescence imaging analysis system (Tanon, Shanghai, China). The images were semiquantified using ImageJ software.

Luciferase activity assay The Egr1 wild-type luciferase reporter plasmid and mutant-type luciferase reporter plasmid were purchased from Kidan Biosciences (Guangzhou, China). One hundred nanograms of luciferase reporter plasmid and $50 \mathrm{nmol}$ of miR-23a-3p mimic were mixed to transfect $293 \mathrm{~T}$ cells, which were seeded in a 96-well plate. Luciferase activity was measured with a Dual-Lumi ${ }^{\mathrm{TM}}$ Luciferase Reporter Gene Assay Kit (Beyotime, Shanghai, China).

Cell viability assays (CCK8 assay) The Cell Counting Kit- 8 (CCK-8) was purchased from Dojindo Molecular Technologies (Kumamoto, Japan). HK-2 cells were seeded in 96-well plates and stimulated with BSA for $48 \mathrm{~h}$. After the stimulation, $10 \mu \mathrm{L}$ CCK-8 solution was added to each well. The optical density at $450 \mathrm{~nm}\left(\mathrm{OD}_{450} \mathrm{~nm}\right)$ was measured after the 96-well plates were incubated for $4 \mathrm{~h}$.

Relative cell viability $=\left(\left[\mathrm{OD}_{\mathrm{BSA}}-\mathrm{OD}_{\text {blank }}\right] /\left[\mathrm{OD}_{\text {control }}-\right.\right.$ $\left.\left.\mathrm{OD}_{\text {blank }}\right]\right) \times 100 \%$.

Statistical analysis All data are presented as the means \pm SEM. SPSS 25.0 software was used to analyze data. Student's $t$-test was used to compare statistical significance between two independent groups. One-way ANOVA was used to determine statistical significance in three or more independent groups. Differences with $P<0.05$ were considered statistically significant.

\section{Results}

The expression of Egr1, IL-6, TNF-a, and FN is increased in DKD To explore the relationship between Egr1 and renal inflammation and fibrosis in DKD, we first detected the expression of Egr1, inflammatory cytokines, and the fibrotic marker in renal tissues of DKD mice. The mRNA levels of Egr1, IL-6, TNF- $\alpha$, and FN were significantly increased as shown by
Table 1. Sequences of the primers used for qRT-PCR in this study

\begin{tabular}{lll}
\hline Genes & Sequences & \\
\hline Egr1 & Sense & \\
& Antisense & 5'-CTGACCGCAGAGTCTTTTCCTG-3' \\
IL-6 & Sense & 5'-TGGGTGCCGCTGAGTAAATG-3' \\
& Antisense & 5'-CAATAACCACCCCTGACC-3' \\
TNF- $\alpha$ & Sense & 5'-GCGCAGAATGAGATGAGTT-3' \\
& Antisense & 5'-GGAAAGGACACCATGAGC-3' \\
FN & Sense & 5'-CCACGATCAGGAAGGAGA-3' \\
& Antisense & 5'-TGGAGAGACAGGAGGAAATAGC-3' \\
$\beta-A c t i n$ & Sense & 5'-CAGTGACAGCATACAGGGTGAT-3' \\
& Antisense & 5'-CCCTGGACTTCGAGCAAGAGAT-3' \\
miR-23a-3p & Sense & 5'-GTTTTCTGCGCAAGTTAGG-3' \\
& Antisense & 5'-ATCACATTGCCAGGGATTTCC-3' \\
U6 & Sense & Universal reverse primer (Tiangen, Beijing, China) \\
& Antisense & 5'-CTCGCTTCGGCAGCACA-3' \\
& & Universal reverse primer (Tiangen, Beijing, China) \\
\hline
\end{tabular}


qRT-PCR (Fig. 1A). And the Western blot results showed that the protein levels of Egr1, TNF- $\alpha$, and FN significantly increased in the renal cortex of mice in the DKD group compared to the control group (Supplementary Fig. 1). In addition, immunohistochemistry analyses revealed that Egr1, IL-6, $\mathrm{TNF}-\alpha$, and FN were significantly increased in the renal tissues in the DKD mice in our previous studies (Xue et al. 2018; Yang et al. 2019; Li et al. 2020). In our previous work, we

A

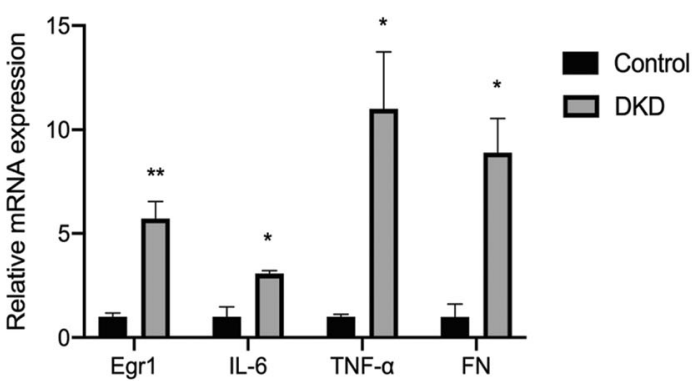

C

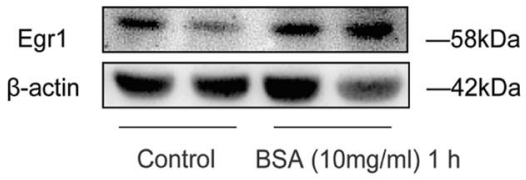

$E$

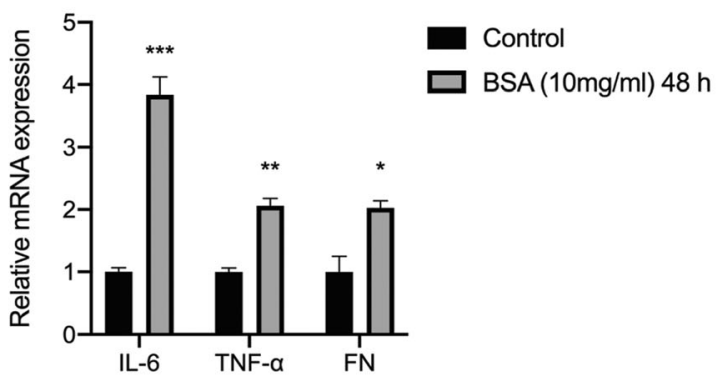

G

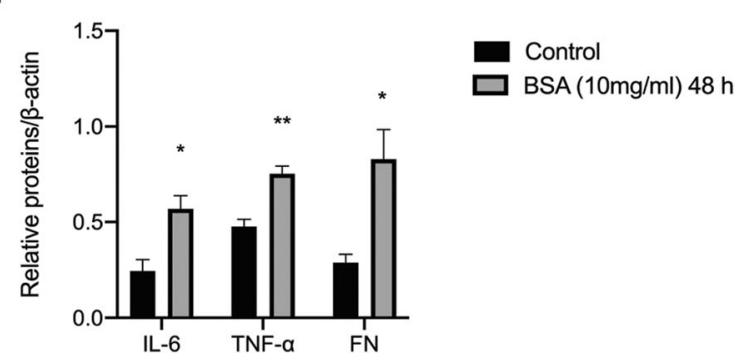

Fig. 1. Elevated expression levels of Egr1, inflammatory cytokines, and fibrosis-related genes in HFD- and STZ-induced DKD mice and BSAinduced HK-2 cells. $A$ The levels of Egr1, IL-6, TNF- $\alpha$, and FN in HFDand STZ-induced DKD mice were quantified by qRT-PCR. $B-D$ Egr1 expression in HK-2 cells cultured in $10 \mathrm{mg} / \mathrm{mL}$ BSA for $1 \mathrm{~h}$ was found that stimulation of PTECs with $10 \mathrm{mg} / \mathrm{mL}$ of BSA for $48 \mathrm{~h}$ induced a fibrotic phenotype which was manifested by increased expression levels of the fibrosis markers TGF- $\beta 1$, wave proteins, and $\alpha$-SMA (Yang et al. 2020). Therefore, we used $10 \mathrm{mg} / \mathrm{mL}$ BSA to stimulate cells to establish an in vitro model of DKD. Our previous work showed that Egr1 increased most significantly after HK-2 cells were stimulated for $1 \mathrm{~h}$ (Xu et al. 2017). Similar to the results of our previous

B

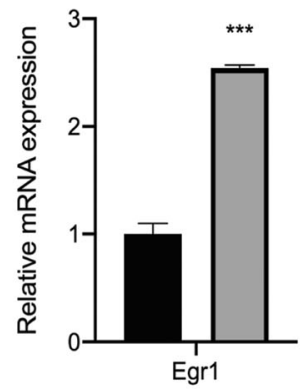

Control
$\square B S(10 \mathrm{mg} / \mathrm{ml}) 1 \mathrm{~h}$

D
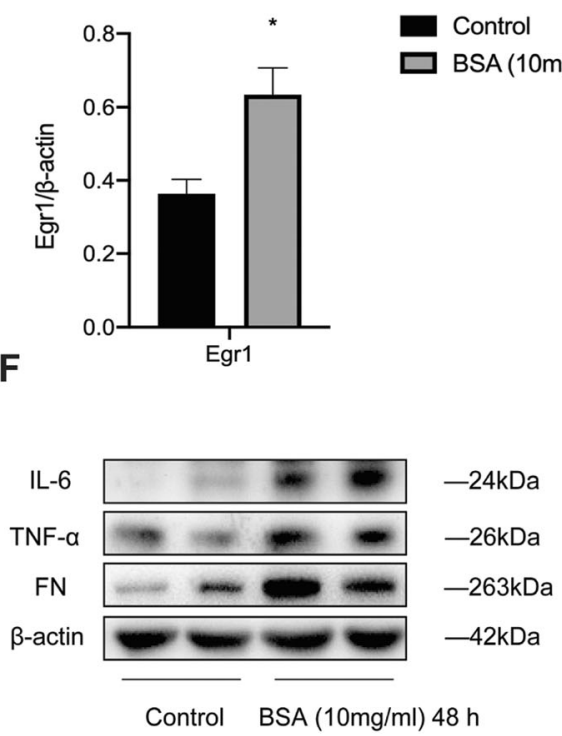

measured by qRT-PCR $(B)$ and Western blot analysis $(C$ and $D) . E-G$ The levels of IL-6, TNF- $\alpha$, and FN in BSA-induced HK-2 cells were detected by qRT-PCR $(E)$ and Western blot analysis $(F$ and $G$ ). Student's $t$-test was used to analyze the statistical significance. Data are reported as the mean \pm SEM. $* P<0.05$, $* * P<0.01$, and $* * * P<0.001$. 
work, we observed a significant increase in Egrl at the mRNA and protein levels (Fig. $1 B-D$ ) in the group treated with BSA for $1 \mathrm{~h}$. The qRT-PCR results also showed that BSA could significantly increase the expression of IL-6, TNF- $\alpha$, and FN (Fig. 1E). Western blot analysis revealed that the expression of IL-6, TNF- $\alpha$, and FN increased in the BSA-treated group (Fig. $1 F$ and $G$ ). Moreover, we found a significant decrease in cell viability of HK-2 cells after exposure to BSA for $48 \mathrm{~h}$ (Supplementary Fig. 2). Thus, we found that the expression levels of Egr1, inflammatory factors, and the fibrotic marker significantly increased both in vivo and in vitro during DKD.

Egr1 promotes the expression of IL-6, TNF- $a$, and FN in BSAinduced HK-2 cells Increased inflammation is a vital mechanism in the progression of DKD. We measured IL- 6 , TNF- $\alpha$, and FN after HK-2 cells were transiently transfected with siEgr1. Egr1 expression was successfully suppressed (Fig. 2A). Egr1 silencing downregulated the expression of IL-6, TNF- $\alpha$, and FN (Fig. 2B). We found that there were consistent trends in protein expression levels and mRNA expression levels (Fig. $2 C$ and $D$ ). Similarly, after transfection with the pENTER-Egr1 plasmid, we found that Egr1 overexpression significantly increased the expression levels of IL-6, TNF- $\alpha$, and FN (Fig. 2E-H). These findings suggest that Egr1 can promote the expression levels of inflammatory factors and the fibrotic marker in HK-2 cells.

miR-23a-3p directly targets Egr1 To further explore the possible mechanism by which Egrl regulates renal inflammation and fibrosis, we used a publicly available algorithm (TargetScan, Cambridge, MA www.targetscan.org/) to identify which miRNAs could target the Egrl 3' UTR. miR23a-3p has been reported to play a vital role in inflammation. miRNA microarray analysis showed that the expression of miR-23a was significantly reduced in endothelial cells treated with TNF- $\alpha$ (Ruan et al. 2012). Another study demonstrated that miR-23a-3p directly inhibits the expression of Bcl-2 family molecules to mitigate neuronal cell death (Sabirzhanov et al. 2020). Therefore, miR-23a-3p, which has a potential target in the 3' UTR of Egr1, was selected as a candidate. Then, we studied the role of miR-23a-3p in DKD. First, we detected the expression level of miR-23a-3p in the kidneys of DKD mice. Compared with that in the control group, miR23a-3p in the kidneys of the mice with DKD was significantly downregulated (Fig. 3A). Similarly, after BSA stimulation, qRT-PCR showed that the expression of miR-23a-3p was significantly decreased in HK-2 cells (Fig. $3 B$ ). The expression levels of miR-23a-3p and Egrl showed the opposite trend both in vivo and in vitro. Next, we determined whether overexpression and silencing of miR-23a-3p could impact Egr1 expression. We used a miR-23a-3p mimic and a miR-23a-3p inhibitor to transfect HK-2 cells. The results showed that the expression of miR-23a-3p was significantly upregulated in
HK-2 cells transfected with the mimic (Fig. 3C) and that the expression of miR-23a-3p in HK-2 cells transfected with the inhibitor was significantly decreased (Fig. $3 G$ ). qRT-PCR and Western blot results revealed that the miR-23a-3p mimic significantly reduced Egr1 expression and that the miR-23a-3p inhibitor increased Egrl expression, respectively (Fig. $3 D-F$ and $H-J$ ).

Next, to investigate the direct regulation of Egrl by miR23a-3p, we constructed luciferase plasmids. One plasmid contained the sequence of the Egr1 3' UTR. The other contained the mutant sequence of the Egrl 3' UTR (Fig. $3 K)$. We cotransfected BSA-treated $293 \mathrm{~T}$ cells with the miR-23a-3p mimic and luciferase plasmids. A dual luciferase reporter assay showed that the luciferase activity in the Egr1 wild-type (WT) plasmid group was inhibited by the miR-23a$3 p$ mimic, while there was no significant change in the luciferase activity in the Egr1 mutant (MUT) plasmid group (Fig. $3 L$ ). In summary, these data suggest that miR-23a-3p directly targets the 3' UTR of Egr1.

Role of miR-23a-3p in regulating the expression of IL-6, TNF$a$, and FN in BSA-treated HK-2 cells To clarify the effect of miR-23a-3p on the inflammatory cytokines IL-6 and TNF- $\alpha$, as well as the fibrotic indicator FN in DKD, we transfected HK-2 cells with the miR-23a-3p mimic and the miR-23a-3p inhibitor and evaluated the effect by qRTPCR and Western blot analysis. The results showed that the mRNA and protein levels of IL- 6 , TNF- $\alpha$, and FN were significantly downregulated in the HK-2 cells transfected with the mimic (Fig. $4 A-C$ ). In contrast, the mRNA and protein levels of IL-6, TNF- $\alpha$, and FN were markedly increased in the HK-2 cells transfected with the inhibitor (Fig. $4 D-F$ ). These results indicate that miR-23a-3p can ameliorate the expression of inflammatory cytokines and fibrotic markers in BSA-stimulated HK-2 cells.

miR-23a-3p alleviates the expression of inflammatory cytokines and fibrotic markers in HK-2 cells via Egr1 To demonstrate whether miR-23a-3p regulates renal inflammation and fibrosis through Egr1, we cotransfected BSA-treated HK-2 cells with a miR-23a-3p inhibitor and si-Egr1. qRT-PCR and Western blot analysis revealed that the miR-23a-3p inhibitor induced upregulation of IL-6, TNF- $\alpha$, and FN. These effects were restored by si-Egr1 transfection (Fig. 5A-C). These data demonstrate that miR-23a-3p regulates the expression of inflammatory cytokines and fibrotic indicators in HK2 cells through Egr1.

\section{Discussion}

In our study, we demonstrated an important role of the miR23a-3p/Egrl pathway in DKD. Our evidence showed that the 
A

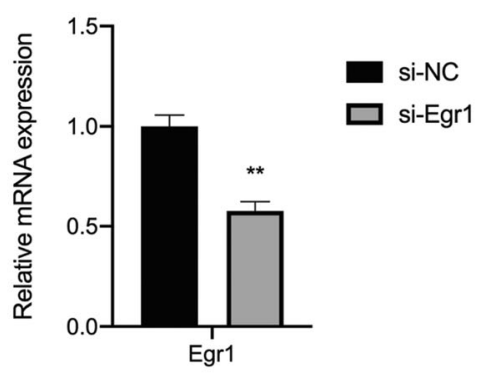

C

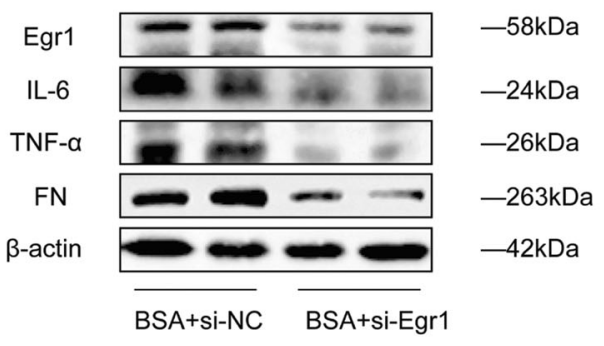

E

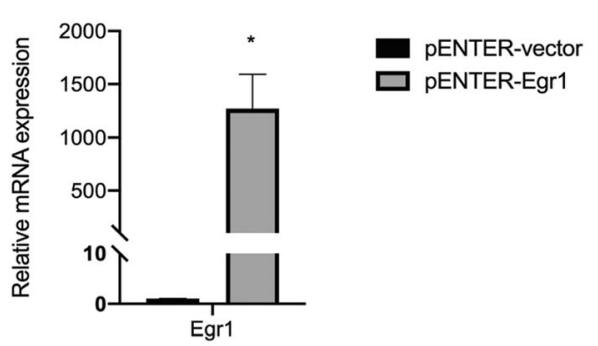

G

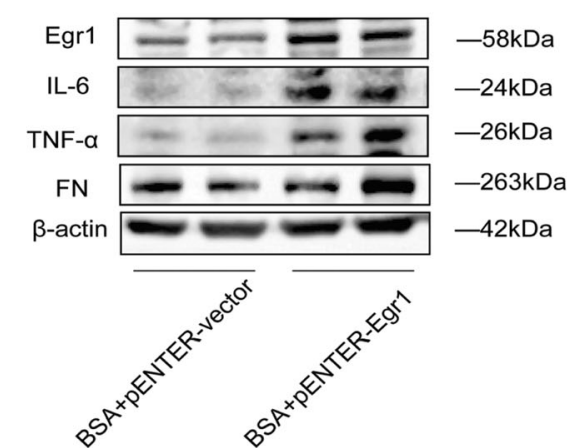

Fig. 2. Egr1 increased the expression levels of inflammatory cytokines and fibrosis-related genes in BSA-induced HK-2 cells. $(A)$ The efficiency of si-Egr1 was determined by qRT-PCR. $(B-D)$ The mRNA and protein expression levels of IL- 6 , TNF- $\alpha$, and FN were detected in BSA-induced HK-2 cells transfected with si-Egr1 for $48 \mathrm{~h}$. $(E)$ The efficiency of pENTER-Egrl was determined by qRT-PCR. $(F-H)$ The mRNA and

expression of miR-23a-3p was decreased in DKD. In addition, we found that miR-23a-3p can alleviate the inflammatory response and the expression of fibrotic markers in HK-2 cells by inhibiting Egr1 expression.

B

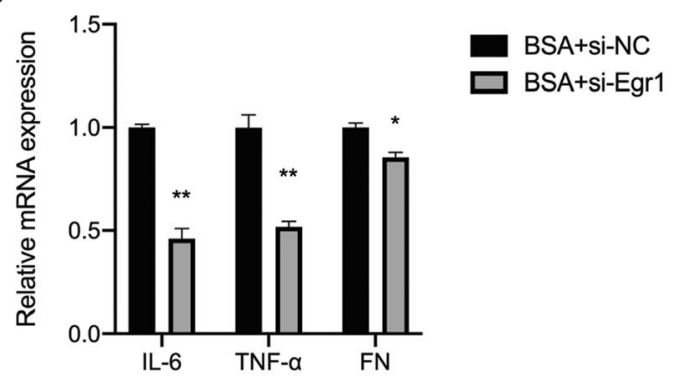

D

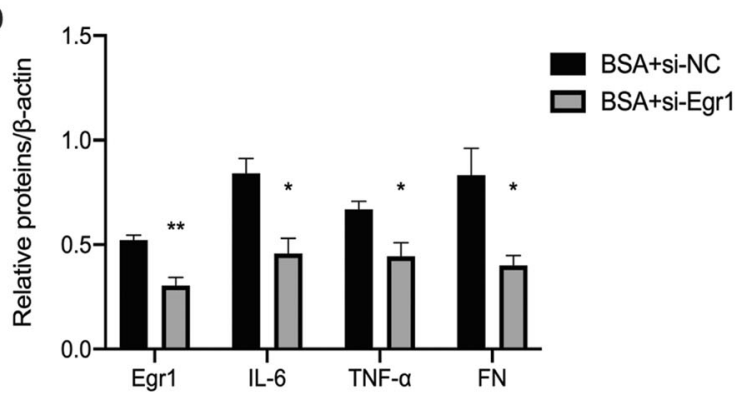

$\mathbf{F}$

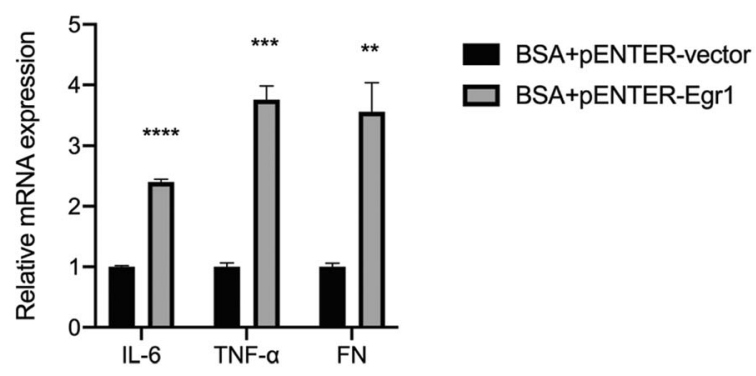

H

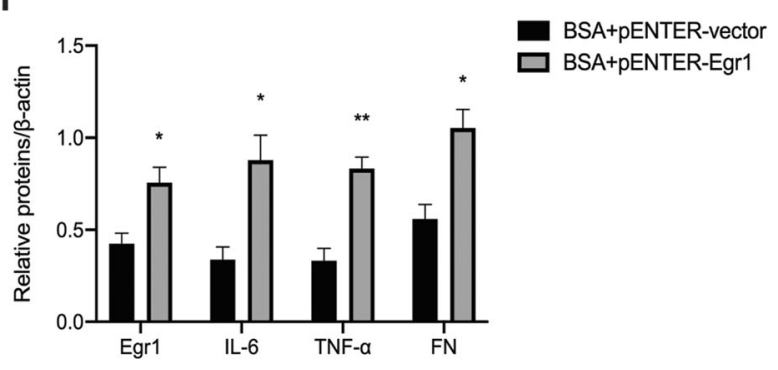

protein expression levels of IL-6, TNF- $\alpha$, and FN were detected in BSA-induced HK-2 cells transfected with pENTER-Egr1 for $48 \mathrm{~h}$. Student's $t$-test was used to analyze the statistical significance. Data are reported as the mean \pm SEM. $* P<0.05, * * P<0.01, * * * P<0.001$, and $* * * * P<0.0001$.

Proteinuria is generally considered one of the biomarkers of early kidney damage and an independent predictor of progressive kidney damage (Slyne et al. 2015; Liew et al. 2020). Several studies have shown that 
A

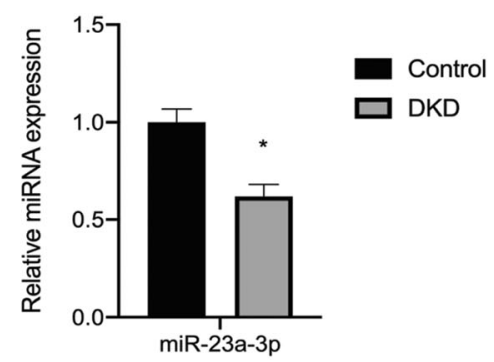

D

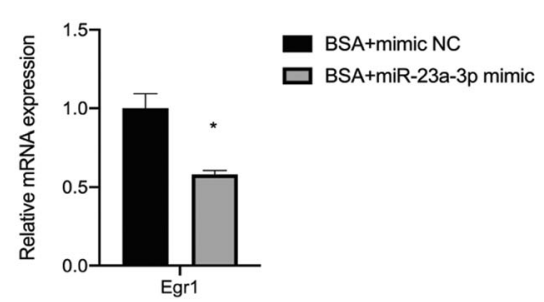

G

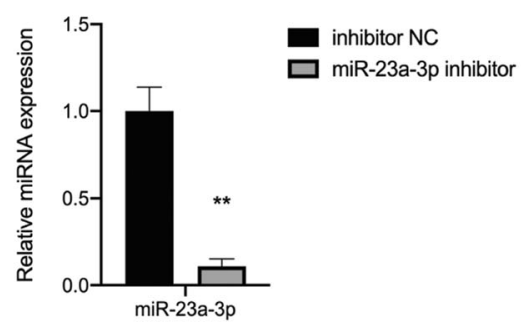

$J$

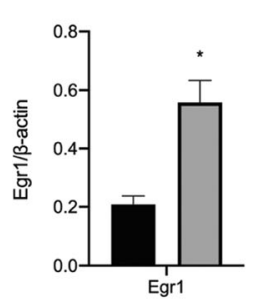

BSA+inhibitor NC

$\square$ BSA+miR-23a-3p inhibitor
B

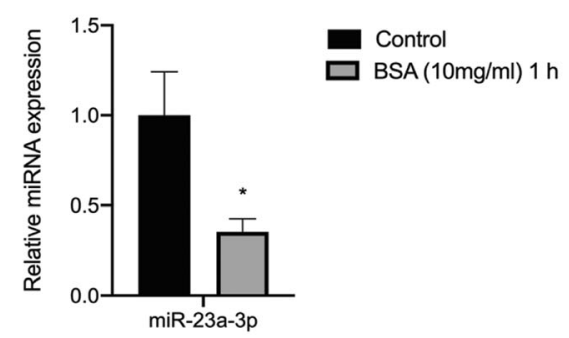

$\mathbf{E}$

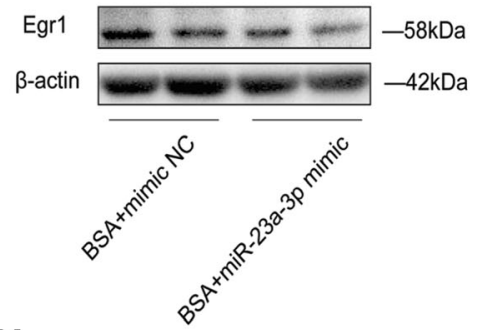

H

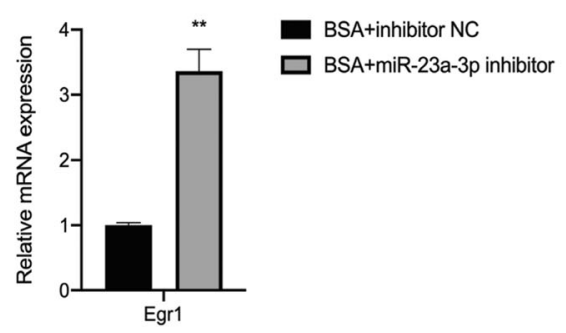

K

5'...UUGGGGUACUCUUGAUGUGAAG...3' position WT-446-452 of Egr1 3'UTR

|||| $\mid$

3' CCUUUAgggaccGuUACACUA 5 ' miR-23a-3p

|| |||

5'...UUGgGgUACUCUUGAUGUGAAG...3' position MUT-446-452 of Egr1 3'UTR

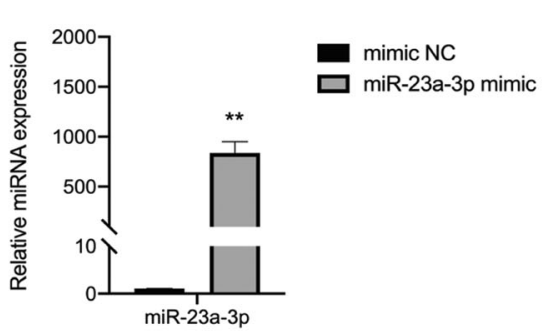

$\mathbf{F}$

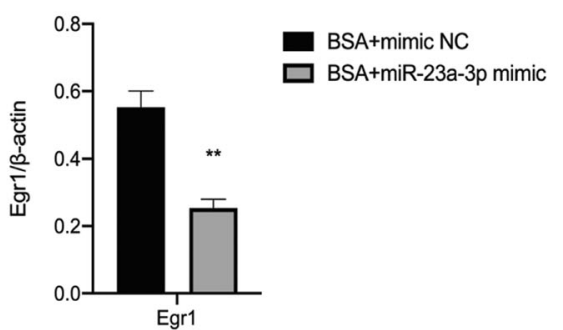

I

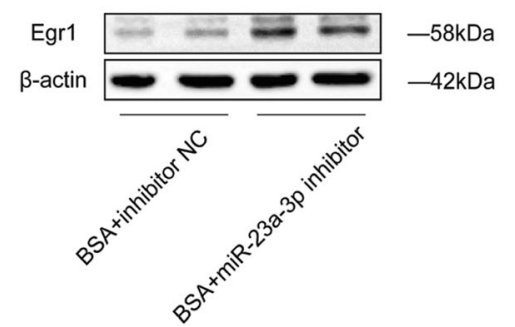

$\mathbf{L}$

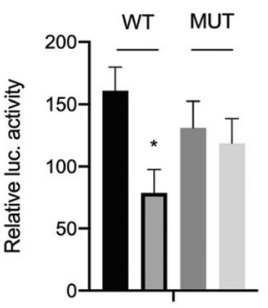

$\operatorname{mimic} \mathrm{NC}+-+$
Fig. 3. The Egr1 3' UTR was regulated by miR-23a-3p. (A, B) miR-23a$3 \mathrm{p}$ expression was measured by qRT-PCR in DKD mice and BSAinduced HK-2 cells. $(C)$ The efficiency of the miR-23a-3p mimic was determined by qRT-PCR. $(D-F)$ The mRNA and protein expression levels of Egrl were detected in BSA-induced HK-2 cells transfected with the miR-23a-3p mimic for 48 h. $(G)$ The efficiency of the miR-23a-3p inhibitor was determined by qRT-PCR. $(H-J)$ The mRNA and protein expression levels of Egr1 were detected in BSA-induced HK-2 cells

increased proteinuria is associated with the progression of CKD and poor outcomes (Waijer et al. 2020). Previous studies have suggested that the exposure of PTECs to excess proteins induces activation of the inflammatory transfected with the miR-23a-3p inhibitor for $48 \mathrm{~h}$. $(K)$ Putative binding sequence of miR-23a-3p in the $3^{\prime}$ UTR of Egr1. The putative binding sequence was eliminated in mutant-type Egrl $3^{\prime}$ UTR luciferase reporter plasmids. $(L)$ Luciferase assays of $293 \mathrm{~T}$ cells cotransfected with the miR23a-3p mimic combined with wild- or mutant-type Egr1 3' UTR luciferase reporter plasmids. Student's $t$-test was used to analyze the statistical significance. Data are reported as the mean \pm SEM. $* P<0.05$ and $* * P<$ 0.01 .

response and EMT (Tang et al. 2003; Wu et al. 2014). Therefore, it is valuable to explore the specific mechanisms of renal tubular damage caused by proteinuria. In our study, we found that BSA can lead to an upregulation 
A

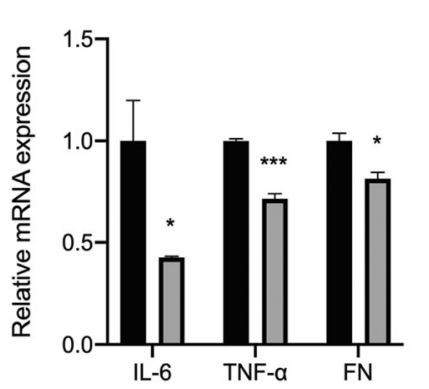

C

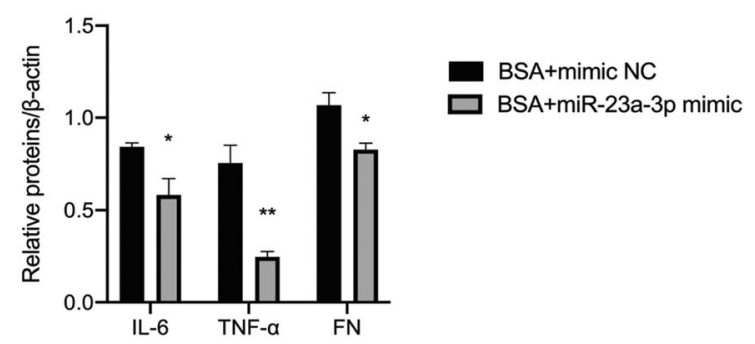

E

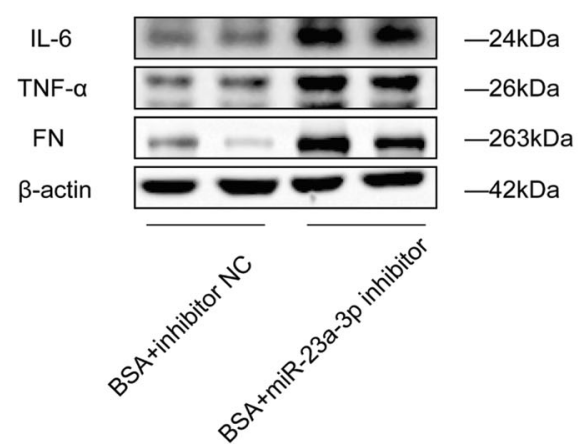

Fig. 4. miR-23a-3p regulated the expression levels of inflammatory cytokines and fibrosis-related genes in BSA-induced HK-2 cells. $(A-C)$ The mRNA and protein expression levels of IL- 6 , TNF- $\alpha$, and FN were detected in BSA-induced HK-2 cells transfected with the miR-23a-3p mimic for $48 \mathrm{~h} .(D-F)$ The mRNA and protein expression levels of IL-

of the expression levels of inflammatory cytokines and fibrosis markers in HK-2 cells.

miRNAs are a group of small noncoding RNAs that are involved in many pathophysiological processes (Bartel 2018). miRNAs have been found to regulate diabetes-related metabolism (Grieco et al. 2017; Lozano-Bartolomé et al. 2018; Chang et al. 2020; Garavelli et al. 2020), and growing evidence has demonstrated the critical role of miRNAs in DKD, highlighting their potential as targets for the treatment of diabetes and its complications. For example, it has been reported
B

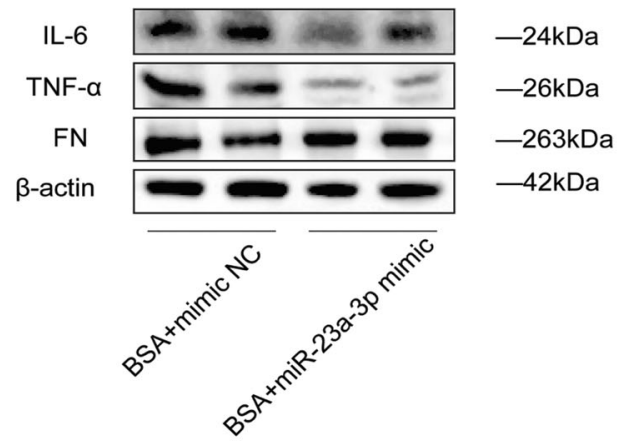

D

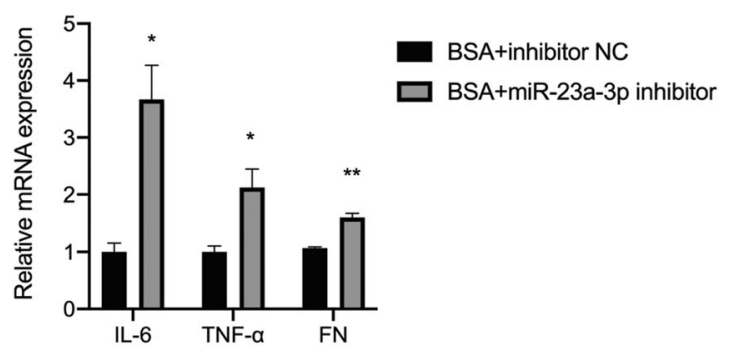

$\mathbf{F}$

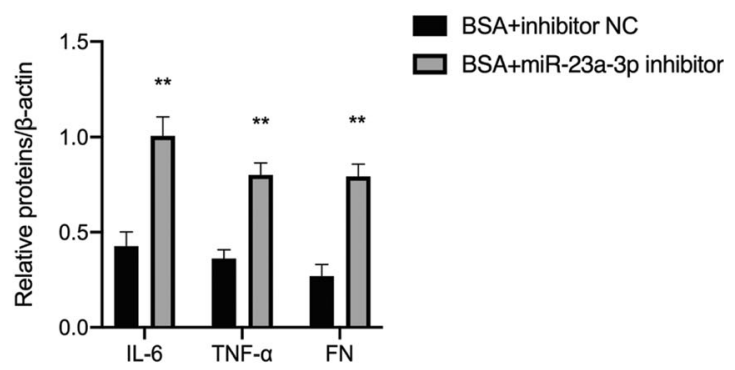

6, TNF- $\alpha$, and FN were detected in BSA-induced HK-2 cells transfected with the miR-23a-3p inhibitor for $48 \mathrm{~h}$. Student's $t$-test was used to analyze the statistical significance. Data are reported as the mean \pm SEM. $* P$ $<0.05, * * P<0.01$, and $* * * P<0.001$.

that miR-4756 aggravates EMT and endoplasmic reticulum stress in DKD via Sestrin2 (Jia et al. 2019). Previous studies have reported that miR-26a and miR-30c play a protective role in DKD through connective tissue growth factor (Zheng et al. 2016). Other miRNAs involved in DKD include miR-192, miR-215, miR-21, miR-29a, miR-181a, and miR-1207-5p (Yarahmadi et al. 2020). Increasing evidence suggests that inflammation and fibrosis are the main features associated with the progression of DKD (Wada and Makino 2016). Recent studies have reported the active involvement of the 
Fig. 5. miR-23a-3p regulated BSA-induced HK-2 cell injury through Egr1. HK-2 cells were transfected with the miR-23a-3p inhibitor and si-Egr1 for 48 h. (A) The mRNA levels of IL-6, TNF$\alpha$, and FN were detected by qRTPCR. $(B, C)$ The protein levels of IL- 6 , TNF- $\alpha$, and FN were detected by Western blot analysis. One-way ANOVA was used to compare three or more independent groups, the Bonferroni test was used for homogeneous variances, and Dunnett's T3-test was used for heterogeneous variances. Data are reported as the mean \pm SEM. $* P<0.05$ and $* * P<0.01$ vs $\mathrm{BSA}+$ inhibitor $\mathrm{NC}+\mathrm{si}-\mathrm{NC}$ group; $\# P<0.05, \# \# P<0.01$,

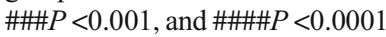
vs BSA + miR-23a-3p inhibitor + si-NC group.
A

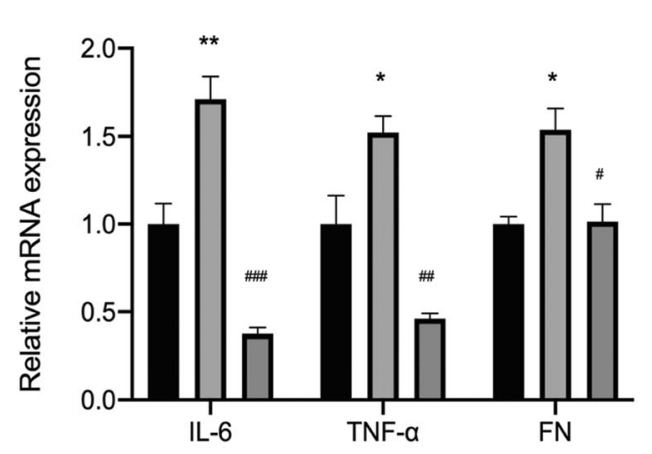

B

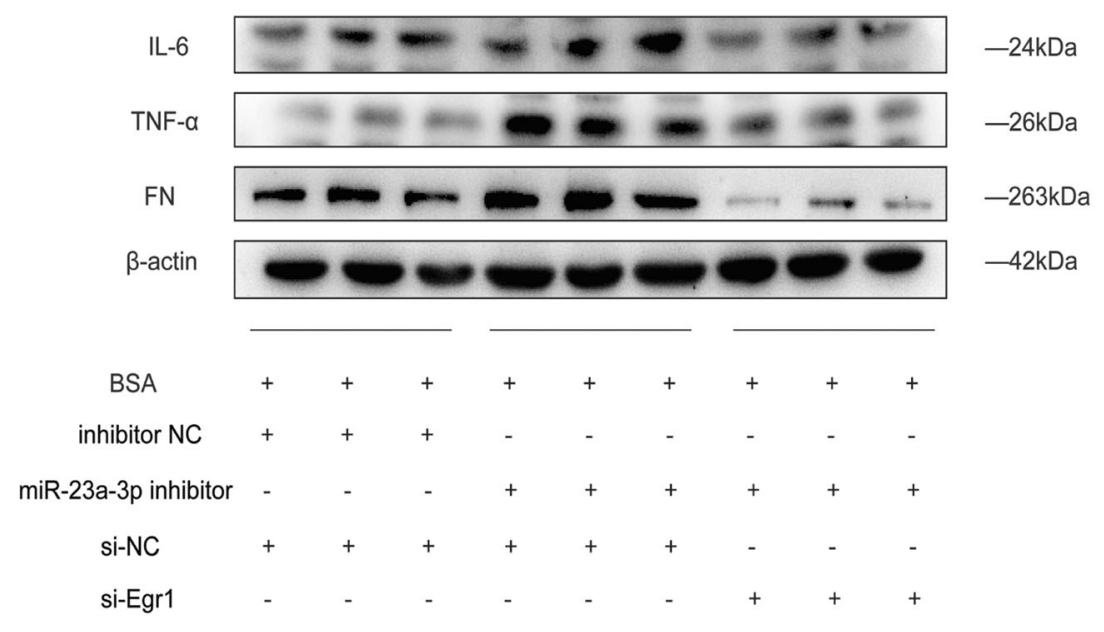

C

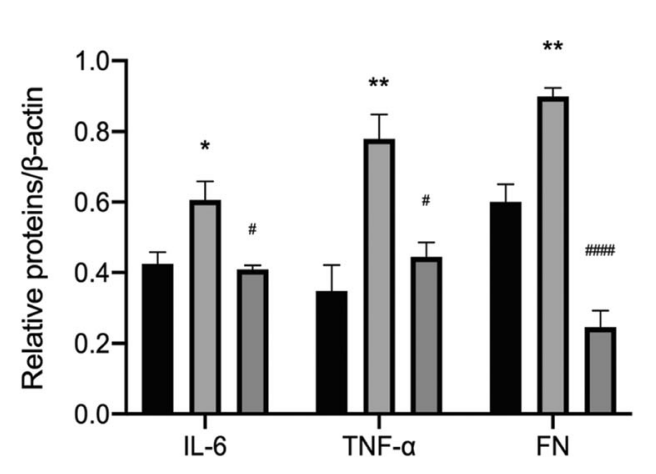

$\mathbf{B}$

BSA+inhibitor NC+si-NC

$\square$ BSA+miR-23a-3p inhibitor+si-NC

$\square$ BSA+miR-23a-3p inhibitor+si-Egr1
$\mathrm{BSA}+$ inhibitor $\mathrm{NC}+\mathrm{si}-\mathrm{NC}$

BSA+miR-23a-3p inhibitor+si-NC

$B S A+m i R-23 a-3 p$ inhibitor+si-Egr1
miR-23a family in regulating the inflammatory response. For example, a study reported that the downregulation of miR-23a was the most significant among a group of miRNAs that were consistently downregulated following LPS stimulation by clustering analysis on the GEO dataset (Si et al. 2018). miR23a was found to play an important role in the development of type 2 diabetes (T2DM) (de Candia et al. 2017). It has been reported that miR-23a-3p is associated with TNF- $\alpha$-induced insulin resistance (Lozano-Bartolomé et al. 2018). Additionally, a previous study showed that serum miR-23a levels were reduced in patients with T2DM (Yang et al.
2014). Moreover, miR-23a-3p was shown to inhibit monocyte function and phagocytosis by targeting IRF1/SP1 followed by the TLR4/TNF- $\alpha /$ TGF- $\beta 1 /$ IL-10 signaling pathway in patients with active tuberculosis (Chen et al. 2020). However, the specific mechanisms of miR-23a-3p in the pathogenesis of DKD have not yet been reported. Therefore, we explored the role of miR-23a-3p in DKD. In this study, miR-23a-3p was significantly decreased in the kidneys of DKD mice. In vitro, miR-23a-3p was decreased in the BSA-treated HK-2 cells. Our results also indicated that inhibition of miR-23a-3p significantly accelerated the expression of inflammatory 
cytokines and fibrotic indicators in HK-2 cells. These findings indicated that miR-23a-3p may be one of the crucial miRNAs involved in the progression of DKD.

Egrl plays a profibrotic role in DKD. One previous study published by our research team showed that miR-181a-5p could decrease the expression of profibrotic genes by suppressing Egr1 (Xu et al. 2017). Furthermore, we found that Egr1 exacerbated the progression of DKD by promoting ECM production, which depended on the long noncoding RNA Arid2-IR (Yang et al. 2019). The potential mechanism by which Egrl affects renal tubular injury still needs to be investigated. As a proinflammatory transcription factor, Egr1 has also been widely studied in a variety of disease models. Egr1 can directly target TGF- $\beta$ to regulate downstream inflammation (Havis and Duprez 2020). On the other hand, Egr1 can also directly act on the promoter of proinflammatory cytokines (such as TNF- $\alpha$ ) to regulate their expression (Bhattacharyya et al. 2011). In another study, Egr1 also regulated endotoxin-triggered NF- $\mathrm{KB}$ signaling by inducing PPAR $\gamma$ (Do et al. 2012). In this study, we also found that Egr1 silencing significantly improved the expression of inflammatory factors and fibrotic indicators in BSA-treated HK-2 cells. Conversely, Egr1 overexpression exacerbated the development of DKD. Our results confirmed the importance of Egr1 in the progression of DKD.

Besides, it is necessary to explore the role of miR-23a-3p in vivo by establishing mouse models with DKD treated with miR-23a-3p agomir or antagomir for further studies. And we need to avoid off-target effect via structural motifs, sequence selection, and chemical formulation of RNA interference triggers when applying synthetic agomirs or antagomirs in vivo (Sarvestani et al. 2015; Bartoszewski and Sikorski 2019; Setten et al. 2019).

\section{Conclusion}

Together, we demonstrated a protective role of miR-23a-3p in albumin-induced HK-2 cells. miR-23a-3p attenuates the inflammatory response and fibrosis by inhibiting the expression of Egr1 in DKD, suggesting that miR-23a-3p may be a new target for the treatment of DKD. The significance and application of miR-23a-3p in the diagnosis and treatment of this disease deserve further exploration.

Supplementary Information The online version contains supplementary material available at https://doi.org/10.1007/s11626-021-00606-1.

Funding This work was supported by research grants from the National Natural Science Foundation of China (Grant No. 81870570 and Grant No. 82070848).

\section{Declarations}

Conflict of interest The authors declare no competing interests.

Open Access This article is licensed under a Creative Commons Attribution 4.0 International License, which permits use, sharing, adaptation, distribution and reproduction in any medium or format, as long as you give appropriate credit to the original author(s) and the source, provide a link to the Creative Commons licence, and indicate if changes were made. The images or other third party material in this article are included in the article's Creative Commons licence, unless indicated otherwise in a credit line to the material. If material is not included in the article's Creative Commons licence and your intended use is not permitted by statutory regulation or exceeds the permitted use, you will need to obtain permission directly from the copyright holder. To view a copy of this licence, visit http://creativecommons.org/licenses/by/4.0/.

\section{References}

Bartel DP (2018) Metazoan microRNAs. Cell 173:20-51

Bartoszewski R, Sikorski AF (2019) Editorial focus: understanding offtarget effects as the key to successful RNAi therapy. Cell Mol Biol Lett 24:69

Bhattacharyya S, Chen SJ, Wu M, Warner-Blankenship M, Ning H, Lakos G, Mori Y, Chang E, Nihijima C, Takehara K, FeghaliBostwick C, Varga J (2008) Smad-independent transforming growth factor-beta regulation of early growth response-1 and sustained expression in fibrosis: implications for scleroderma. Am J Pathol 173:1085-1099

Bhattacharyya S, Wu M, Fang F, Tourtellotte W, Feghali-Bostwick C, Varga J (2011) Early growth response transcription factors: key mediators of fibrosis and novel targets for anti-fibrotic therapy. Matrix Biol 30:235-242

Broughton JP, Lovci MT, Huang JL, Yeo GW, Pasquinelli AE (2016) Pairing beyond the seed supports microRNA targeting specificity. Mol Cell 64:320-333

Chang H, Chang H, Cheng T, Lee GD, Chen X, Qi K (2021) Microribonucleic acid-23a-3p prevents the onset of type 2 diabetes mellitus by suppressing the activation of nucleotide-binding oligomerization-like receptor family pyrin domain containing 3 inflammatory bodies-caused pyroptosis through negatively regulating NIMA-related kinase 7. J Diabetes Investig 12:334-345

Chen SJ, Ning H, Ishida W, Sodin-Semrl S, Takagawa S, Mori Y, Varga J (2006) The early-immediate gene EGR-1 is induced by transforming growth factor-beta and mediates stimulation of collagen gene expression. J Biol Chem 281:21183-21197

Chen YC, Lee CP, Hsiao CC, Hsu PY, Wang TY, Wu CC, Chao TY, Leung SY, Chang YP, Lin MC (2020) MicroRNA-23a-3p DownRegulation in Active Pulmonary Tuberculosis Patients with High Bacterial Burden Inhibits Mononuclear Cell Function and Phagocytosis through TLR4/TNF- $\alpha /$ TGF- $\beta 1 /$ IL-10 Signaling via Targeting IRF1/SP1. Int J Mol Sci 21:8587

de Candia P, Spinetti G, Specchia C, Sangalli E, La Sala L, Uccellatore A, Lupini S, Genovese S, Matarese G, Ceriello A (2017) A unique plasma microRNA profile defines type 2 diabetes progression. PLoS One 12:e0188980

Do KH, Choi HJ, Kim J, Park SH, Kim HH, Oh CG, Moon Y (2012) Ambivalent roles of early growth response 1 in inflammatory signaling following ribosomal insult in human enterocytes. Biochem Pharmacol 84:513-521

Eller K, Kirsch A, Wolf AM, Sopper S, Tagwerker A, Stanzl U, Wolf D, Patsch W, Rosenkranz AR, Eller P (2011) Potential role of regulatory $\mathrm{T}$ cells in reversing obesity-linked insulin resistance and diabetic nephropathy. Diabetes 60:2954-2962 
International Diabetes Federation. IDF Diabetes Atlas: 9th Edition 2019 (Online). http://www.diabetesatlas.org

Garavelli S, Bruzzaniti S, Tagliabue E, Di Silvestre D, Prattichizzo F, Mozzillo E, Fattorusso V, La Sala L, Ceriello A, Puca AA, Mauri P, Strollo R, Marigliano M, Maffeis C, Petrelli A, Bosi E, Franzese A, Galgani M, Matarese G, de Candia P (2020) Plasma circulating miR-23 27 24 clusters correlate with the immunometabolic derangement and predict $\mathrm{C}$-peptide loss in children with type 1 diabetes. Diabetologia 63:2699-2712

Grieco FA, Sebastiani G, Juan-Mateu J, Villate O, Marroqui L, Ladrière L, Tugay K, Regazzi R, Bugliani M, Marchetti P, Dotta F, Eizirik DL (2017) MicroRNAs miR-23a-3p, miR-23b-3p, and miR-149-5p regulate the expression of proapoptotic $\mathrm{BH} 3$-only proteins DP5 and PUMA in human pancreatic $\beta$-cells. Diabetes 66:100-112

Hameed I, Masoodi SR, Malik PA, Mir SA, Ghazanfar K, Ganai BA (2018) Genetic variations in key inflammatory cytokines exacerbates the risk of diabetic nephropathy by influencing the gene expression. Gene 661:51-59

Havis E, Duprez D (2020) EGR1 transcription factor is a multifaceted regulator of matrix production in tendons and other connective tissues. Int J Mol Sci 21:1664

Heerspink H, Greene T, Tighiouart H, Gansevoort RT, Coresh J, Simon AL, Chan TM, Hou FF, Lewis JB, Locatelli F, Praga M, Schena FP, Levey AS, Inker LA (2019) Change in albuminuria as a surrogate endpoint for progression of kidney disease: a meta-analysis of treatment effects in randomised clinical trials. Lancet Diabetes Endocrinol 7:128-139

Hu F, Xue M, Li Y, Jia YJ, Zheng ZJ, Yang YL, Guan MP, Sun L, Xue YM (2018) Early Growth Response 1 (Egr1) Is a transcriptional activator of NOX4 in oxidative stress of diabetic kidney disease. J Diabetes Res 2018:3405695

Hu J, Zhai C, Hu J, Li Z, Fei H, Wang Z, Fan W (2017) MiR-23a inhibited IL-17-mediated proinflammatory mediators expression via targeting IKK $\alpha$ in articular chondrocytes. Int Immunopharmacol 43:1-6

Jia Y, Zheng Z, Guan M, Zhang Q, Li Y, Wang L, Xue Y (2018) Exendin-4 ameliorates high glucose-induced fibrosis by inhibiting the secretion of miR-192 from injured renal tubular epithelial cells. Exp Mol Med 50:1-13

Jia Y, Zheng Z, Yang Y, Zou M, Li J, Wang L, Guan M, Xue Y (2019) MiR-4756 promotes albumin-induced renal tubular epithelial cell epithelial-to-mesenchymal transition and endoplasmic reticulum stress via targeting Sestrin2. J Cell Physiol 234:2905-2915

Li S, Jia Y, Xue M, Hu F, Zheng Z, Zhang S, Ren S, Yang Y, Si Z, Wang L, Guan M, Xue Y (2020) Inhibiting Rab27a in renal tubular epithelial cells attenuates the inflammation of diabetic kidney disease through the miR-26a-5p/CHAC1/NF-kB pathway. Life Sci 261: 118347

Liew A, Bavanandan S, Prasad N, Wong MG, Chang JM, Eiam-Ong S, Hao CM, Lim CY, Lim SK, Oh KH, Okada H, Susantitaphong P, Lydia A, Tran HTB, Villanueva R, Yeo SC, Tang SCW (2020) Asian Pacific Society of Nephrology clinical practice guideline on diabetic kidney disease. Nephrology (Carlton) 25(Suppl 2):12-45

Liu M, Liu SW, Wang LJ, Bai YM, Zeng XY, Guo HB, Liu YN, Jiang YY, Dong WL, He GX, Zhou MG, Yu SC (2019) Burden of diabetes, hyperglycaemia in China from to 2016: findings from the 1990 to 2016, global burden of disease study. Diabetes Metab 45: 286-293

Lozano-Bartolomé J, Llauradó G, Portero-Otin M, Altuna-Coy A, RojoMartínez G, Vendrell J, Jorba R, Rodríguez-Gallego E, Chacón MR (2018) Altered expression of miR-181a-5p and miR-23a-3p is associated with obesity and TNF $\alpha$-induced insulin resistance. J Clin Endocrinol Metab 103:1447-1458

Mohajeri M, Banach M, Atkin SL, Butler AE, Ruscica M, Watts GF, Sahebkar A (2018) MicroRNAs: novel molecular targets and response modulators of statin therapy. Trends Pharmacol Sci 39: 967-981

Peng W, Huang S, Shen L, Tang Y, Li H, Shi Y (2019) Long noncoding RNA NONHSAG053901 promotes diabetic nephropathy via stimulating Egr-1/TGF- $\beta$-mediated renal inflammation. J Cell Physiol 234:18492-18503

Ruan W, Xu JM, Li SB, Yuan LQ, Dai RP (2012) Effects of downregulation of microRNA-23a on TNF- $\alpha$-induced endothelial cell apoptosis through caspase-dependent pathways. Cardiovasc Res 93:623-632

Sabirzhanov B, Makarevich O, Barrett J, Jackson IL, Faden AI, Stoica BA (2020) Down-regulation of miR-23a-3p mediates irradiationinduced neuronal apoptosis. Int J Mol Sci 21:3695

Sakai N, Wada T (2015) Revisiting inflammation in diabetic nephropathy: the role of the Nlrp3 inflammasome in glomerular resident cells. Kidney Int 87:12-14

Sarvestani ST, Stunden HJ, Behlke MA, Forster SC, McCoy CE, Tate MD, Ferrand J, Lennox KA, Latz E, Williams BR, Gantier MP (2015) Sequence-dependent off-target inhibition of TLR7/8 sensing by synthetic microRNA inhibitors. Nucleic Acids Res 43:11771188

Setten RL, Rossi JJ, Han SP (2019) The current state and future directions of RNAi-based therapeutics. Nat Rev Drug Discov 18:421-446

Si X, Cao D, Chen J, Nie Y, Jiang Z, Chen MY, Wu JF, Guan XD (2018) miR-23a downregulation modulates the inflammatory response by targeting ATG12-mediated autophagy. Mol Med Rep 18:15241530

Slyne J, Slattery C, McMorrow T, Ryan MP (2015) New developments concerning the proximal tubule in diabetic nephropathy: in vitro models and mechanisms. Nephrol Dial Transplant 30(Suppl 4): iv60-iv67

Sun L, Kanwar YS (2015) Relevance of TNF- $\alpha$ in the context of other inflammatory cytokines in the progression of diabetic nephropathy. Kidney Int 88:662-665

Tang S, Leung JC, Abe K, Chan KW, Chan LY, Chan TM, Lai KN (2003) Albumin stimulates interleukin-8 expression in proximal tubular epithelial cells in vitro and in vivo. J Clin Invest 111:515-527

Wada J, Makino H (2016) Innate immunity in diabetes and diabetic nephropathy. Nat Rev Nephrol 12:13-26

Waijer SW, Xie D, Inzucchi SE, Zinman B, Koitka-Weber A, Mattheus M, von Eynatten M, Inker LA, Wanner C, Heerspink H (2020) Short-term changes in albuminuria and risk of cardiovascular and renal outcomes in type 2 diabetes mellitus: a post hoc analysis of the EMPA-REG OUTCOME Trial. J Am Heart Assoc 9:e016976

Wang D, Guan MP, Zheng ZJ, Li WQ, Lyv FP, Pang RY, Xue YM (2015) Transcription factor Egr1 is involved in high glucoseinduced proliferation and fibrosis in rat glomerular mesangial cells. Cell Physiol Biochem 36:2093-2107

Wong D, Yiu WH, Chan KW, Li Y, Li B, Lok S, Taketo MM, Igarashi P, Chan L, Leung J, Lai KN, Tang S (2018) Activated renal tubular $\mathrm{Wnt} / \beta$-catenin signaling triggers renal inflammation during overload proteinuria. Kidney Int 93:1367-1383

Wu HJ, Yiu WH, Li RX, Wong DW, Leung JC, Chan LY, Zhang Y, Lian Q, Lin M, Tse HF, Lai KN, Tang SC (2014) Mesenchymal stem cells modulate albumin-induced renal tubular inflammation and fibrosis. PLoS One 9:e90883

Xu P, Guan MP, Bi JG, Wang D, Zheng ZJ, Xue YM (2017) High glucose down-regulates microRNA-181a-5p to increase profibrotic gene expression by targeting early growth response factor 1 in HK-2 cells. Cell Signal 31:96-104

Xue M, Li Y, Hu F, Jia YJ, Zheng ZJ, Wang L, Xue YM (2018) High glucose up-regulates microRNA-34a-5p to aggravate fibrosis by targeting SIRT1 in HK-2 cells. Biochem Biophys Res Commun 498:38-44

Yang YL, Hu F, Xue M, Jia YJ, Zheng ZJ, Li Y, Xue YM (2019) Early growth response protein-1 upregulates long noncoding RNA Arid2- 
IR to promote extracellular matrix production in diabetic kidney disease. Am J Phys Cell Phys 316:C340-C352

Yang YL, Xue M, Jia YJ, Hu F, Zheng ZJ, Wang L, Si ZK, Xue YM (2020) Long noncoding RNA NEAT1 is involved in the protective effect of Klotho on renal tubular epithelial cells in diabetic kidney disease through the ERK1/2 signaling pathway. Exp Mol Med 52: 266-280

Yang Z, Chen H, Si H, Li X, Ding X, Sheng Q, Chen P, Zhang H (2014) Serum miR-23a, a potential biomarker for diagnosis of pre-diabetes and type 2 diabetes. Acta Diabetol 51:823-831

Yarahmadi A, Shahrokhi SZ, Mostafavi-Pour Z, Azarpira N (2020) MicroRNAs in diabetic nephropathy: from molecular mechanisms to new therapeutic targets of treatment. Biochem Pharmacol:114301

Zeni L, Norden A, Cancarini G, Unwin RJ (2017) A more tubulocentric view of diabetic kidney disease. J Nephrol 30:701-717
Zha F, Qu X, Tang B, Li J, Wang Y, Zheng P, Ji T, Zhu C, Bai S (2019) Long non-coding RNA MEG3 promotes fibrosis and inflammatory response in diabetic nephropathy via miR-181a/Egr-1/TLR4 axis. Aging (Albany NY) 11:3716-3730

Zhang L, Long J, Jiang W, Shi Y, He X, Zhou Z, Li Y, Yeung RO, Wang J, Matsushita K, Coresh J, Zhao MH, Wang H (2016) Trends in chronic kidney disease in China. N Engl J Med 375:905-906

Zheng Z, Guan M, Jia Y, Wang D, Pang R, Lv F, Xiao Z, Wang L, Zhang $\mathrm{H}$, Xue Y (2016) The coordinated roles of miR-26a and miR-30c in regulating TGF $\beta 1$-induced epithelial-to-mesenchymal transition in diabetic nephropathy. Sci Rep 6:37492

Zhu S, Pan W, Song X, Liu Y, Shao X, Tang Y, Liang D, He D, Wang H, Liu W, Shi Y, Harley JB, Shen N, Qian Y (2012) The microRNA miR-23b suppresses IL-17-associated autoimmune inflammation by targeting TAB2, TAB3 and IKK- $\alpha$. Nat Med 18:1077-1086 